\title{
Evaluación de la efectividad del fipronil al 1\% e ivermectina al $1 \%$ en el tratamiento de la sarna por Trixacarus caviae en cuyes (Cavia porcellus).
}

\author{
Evaluation of the effectiveness of fipronil $1 \%$ and the ivermectin $1 \%$ in the treatment of scabies by \\ Trixacarus caviae in guinea pigs (Cavia porcellus).
}

\author{
Meylin Huamán ${ }^{1}$; Guillermo Leguia²; Lilia Chauca ${ }^{1}$; Luis Torres³ ${ }^{3}$ Néstor Falcón ${ }^{4}$
}

\section{RESUMEN}

El objetivo del estudio fue evaluar la efectividad del fipronil al 1\% y de la ivermectina al $1 \%$ en el tratamiento de sarna causada por Trixacarus caviae en cuyes infestados naturalmente, durante un periodo de 42 días. Para ello se seleccionaron 21 hembras reproductoras, dividiéndose al azar en tres grupos: (a) tratado con fipronil (vía epicutánea) $1,5 \mathrm{ml} / \mathrm{kg}$. p.v., (b) tratado con ivermectina (vía subcutánea) $0,05 \mathrm{ml} / \mathrm{kg}$. p.v. ambos con cuatro tratamientos a intervalos de 8 días, y (c) sin tratamiento. Cada 8 días se colectó escarificaciones cutáneas de cada animal para determinar el porcentaje de ácaros vivos y se tomaron biopsias de piel de un animal por grupo para la evaluación histopatológica. Al inicio del estudio los cuyes de los tres grupos presentaron $100 \%$ de ácaros vivos, observándose al día 42 post tratamiento, una disminución de ácaros vivos hasta $11,1 \%$ en el grupo tratado con fipronil y hasta $0 \%$ en el grupo tratado con ivermectina. La efectividad que alcanzó el fipronil fue de 95,2\% y la ivermectina 100\%, no encontrándose diferencia significativa entre ellas, pero sí con respecto al grupo control. La evolución de la recuperación histopatológica en ambos grupos tratados fue similar al día 42, observándose ausencia de infiltrado inflamatorio y la mayoría de folículos pilosos eran viables. Se concluye que el fipronil y la ivermectina, ambos al $1 \%$, aplicados en cuatro dosis consecutivas con intervalos de 8 días mostraron un efecto acaricida sobre el T. caviae.

PALABRAS CLAVE: Ivermectina, fipronil, Trixacarus caviae, histopatología.

\section{SUMMARY}

The objective of the study was to evaluate the effectiveness of $1 \%$ fipronil and $1 \%$ ivermectin in the treatment of scabies caused by Trixacarus caviae in naturally infested guinea pigs, for a period of 42 days. For this, 21 breeding females were selected, randomly divided into three groups: (a) treated with fipronil (epicutaneous route) $1.5 \mathrm{ml} /$ kg. p.v., (b) treated with ivermectin (subcutaneous route) $0.05 \mathrm{ml} / \mathrm{kg}$. p.v. both with one treatment at intervals of 8 days until completing 4 treatments per group and (c) without treatment. Every 8 days, skin scarifications were collected per animal to determine the percentage of live mites and skin biopsies of one animal were taken per group for histopathological evaluation. At the beginning of the study, the guinea pigs of the three groups presented $100 \%$ live mites, observing 42 post-treatment days, a decrease in live mites up to $11.1 \%$ in the group treated with

\footnotetext{
${ }^{1}$ Instituto Nacional de Innovación Agraria. Lima, Perú.

${ }^{2}$ Universidad Ricardo Palma. Lima, Perú.

${ }^{3}$ Universidad Alas Peruanas. Lima, Perú.

${ }^{4}$ Universidad Peruana Cayetano Heredia. Lima, Perú.
} 
fipronil and up to $0 \%$ in the group treated with ivermectin. The effectiveness reached by fipronil was $95.2 \%$ and ivermectin $100 \%$, with no significant difference between them, but with respect to the control group. The evolution of histopathological recovery in both treated groups was similar to day 42, observing absence of inflammatory infiltrate, most hair follicles were viable. It is concluded that fipronil and ivermectin both at $1 \%$, applied in four consecutive doses with 8 day intervals showed an acaricide effect on T. caviae.

KEYWORDS: Ivermectin, Fipronil, Trixacarus caviae, histopathology.

\section{INTRODUCCIÓN}

Entre los principales problemas sanitarios que enfrenta la producción de cuyes se encuentra la infestación por Trixacarus caviae, ácaro excavador, sarcoptiforme, el cual es responsable de la sarna en cuyes (Collins, Pope y Griffin, 1986; Klompen, 1992; Hendrix, 1999; Scott, Miller, Griffin, Giovanniello y Jure, 2002; White, Bourdeau y Meredith, 2003). Fue identificado por primera vez en Perú por Levano y Chauca (2008) en un trabajo preliminar en el Instituto Nacional de Innovación Agraria (INIA).

Los cuyes pueden ser portadores asintomáticos de $T$. caviae y bajo condiciones de estrés durante el estado fisiológico en gestación y/o lactancia, hacinamiento, poca ventilación, elevada temperatura, entre otros factores que condicionen un deficiente estado inmunitario, se favorecería la aparición de casos clínicos. Los recién nacidos pueden infectarse y mostrar signos clínicos como rascarse dentro de las primeras 72 horas de vida, y enrojecimiento de la piel entre 3 y 4 semanas después del nacimiento; mientras que el desarrollo de signos clínicos en cobayos en etapa de recría y adulto, tarda de 10 a 50 días (Fuentealba y Hanna, 1996).

La lesión primaria inicial corresponde a una alopecia parcial, la superficie de la piel es de apariencia seca a oleosa, con escamas amarillas y costras. La histopatología de la piel pone de manifiesto marcada hiperqueratosis ortoqueratósica, hiperplasia epidérmica, acantosis, áreas focales de espongiosis epidérmica, necrosis y moderada fibrosis, infiltración inflamatoria eosinofílica y linfocítica en la dermis superficial (Ackerman, 1987; Fuentealba y Hanna, 1996; White et al., 2003).

El tratamiento más usado para el control de sarna por diferentes especies son las Ivermectinas. La droga potencializa los efectos del GABA en la sinapsis mediante la estimulación presináptica de la liberación de GABA. El GABA se une a los receptores del canal de cloro (receptor postsináptico), abriendo el canal de cloro, aumentando la entrada de iones cloro el cual hiperpolariza la membrana neuronal haciéndola menos excitatoria, esto hace más difícil la neurotransmisión de estímulos a los músculos provocando su muerte por parálisis flácida (Sumano y Ocampo, 1997; Adams, 2001; Botana, Landoni y Martín-Jiménez, 2002). La dosis empleada de ivermectina al $1 \%$ es de 0.02 a $0.05 \mathrm{ml} / \mathrm{kg}$ de peso vivo (p.v.), por tres tratamientos consecutivos cada 7 a 10 días, con un periodo de retiro mínimo de 28 a 30 días (Swindle, Laber-Laird y Flecknell, 1996; Richardson, 2000; White et al., 2003; Plumb, 2005).

Actualmente, una alternativa efectiva es el fipronil, que interfiere en la regulación nerviosa a nivel del SNC por inhibición del GABA; bloquea el pasaje de iones cloro a través de los canales de cloro causando la muerte del parásito por hiperexcitación, es altamente específico para invertebrados (Adams, 2001; Plumb, 2005). Asimismo, un estudio preliminar realizado por Levano y Chauca (2008) utilizando fipronil al 1\% estableció la dosis de $1.5 \mathrm{ml} / \mathrm{kg}$. p.v. para cuyes.

El estudio tuvo por objetivo evaluar la eficacia del fipronil al 1\% (aplicación epicutánea) e ivermectina al $1 \%$ (vía subcutánea) como tratamiento de la sarna asociada a infecciones por $T$. caviae en cuyes infestados naturalmente.

\section{MATERIAL Y MÉTODOS}

El estudio experimental se realizó en los laboratorios de Parasitología y Patología Animal de la Facultad de Medicina Veterinaria y Zootecnia de la Universidad Peruana Cayetano Heredia. Los especímenes procedieron de granjas familiarcomerciales de la provincia de Lima, distrito de la Molina y Pachacamac, que trabajaron en procesos de validación de tecnologías generadas en el Instituto Nacional de Innovación Agraria de Perú.

Se seleccionaron hembras reproductoras identificadas con aretes, naturalmente infestadas con T. caviae, con edad promedio de 1 año y nueve meses, 
peso entre 1.3 a $1.6 \mathrm{~kg}$. Los animales experimentales fueron sometidos a un mismo manejo, recibiendo una alimentación mixta, un balanceado con $17 \%$ de proteína y forraje, maíz chala o alfalfa.

Los cuyes que formaron parte del estudio presentaron lesiones de sarna en diferentes partes del cuerpo: región axilar, cervical, inguinal, ventral y miembros posteriores. El grado de lesión de los cuyes se clasificó según su presentación clínica en:

Leve: lesiones que consistían en alopecia focalizada con descamación de la piel.

Moderada: cuadro más profundo con alopecia focalizada a extendida, prurito intenso, descamación de la piel y ligera cantidad de costras.

Grave: alopecia extendida, pústulas, gran cantidad de costras, intenso prurito, liquenificación, fístulas y úlceras abiertas como resultado de la automutilación por el escozor.

La descripción de las lesiones se clasificó en grados del 1 al 4, según el siguiente patrón:

Grado 1: Alopecia parcial en una o dos áreas afectadas, se observa dermatitis inicial.

Grado 2: Tres áreas afectadas, se observa dermatitis moderada y presencia de escamas.

Grado 3: Dermatitis pronunciada, presencia de tres o más áreas afectadas con engrosamiento y ligera cantidad de costras.
Grado 4: Dermatitis costrosa con mayor engrosamiento y presencia de costras en área mayor que el grado anterior y algunas fístulas.

Los animales seleccionados para el estudio fueron aquellos que presentaron los 4 grados de lesión los cuales se distribuyeron en forma aleatoria en 3 grupos: Grupo A: tratados con fipronil al 1\% vía epicutánea, aplicación a la zona dorsal a dosis de $1.5 \mathrm{ml} / \mathrm{kg}$ p.v., 4 tratamientos a intervalos de 8 días.

Grupo B: tratados con ivermectina al $1 \%$ vía subcutánea, aplicación en la región costal a dosis de $0.05 \mathrm{ml} / \mathrm{kg}$ p.v., 4 tratamientos a intervalos de 8 días. Grupo C: control sin tratamiento.

Adicionalmente, se utilizó una plantilla individual con una grafica de la silueta del cuy, en la cual se registró el número de arete, localización topográfica y descripción de las lesiones; así como el registro fotográfico individual.

La detección del ácaro se realizó mediante raspados cutáneos, características morfológicas (figura 1 ( $\mathrm{a}$ y b)) y conteo de ácaros en sus diferentes estadios, sean larvas, ninfas o adultos, vivos y/o muertos. La evaluación clínica de las lesiones se evaluó progresivamente en los días $0,8,16,24,32$ y 42 , post tratamiento. Se obtuvieron biopsias de piel de $4 \mathrm{~mm}$ de diámetro de la parte del tejido afectado de un espécimen representativo por cada grupo.

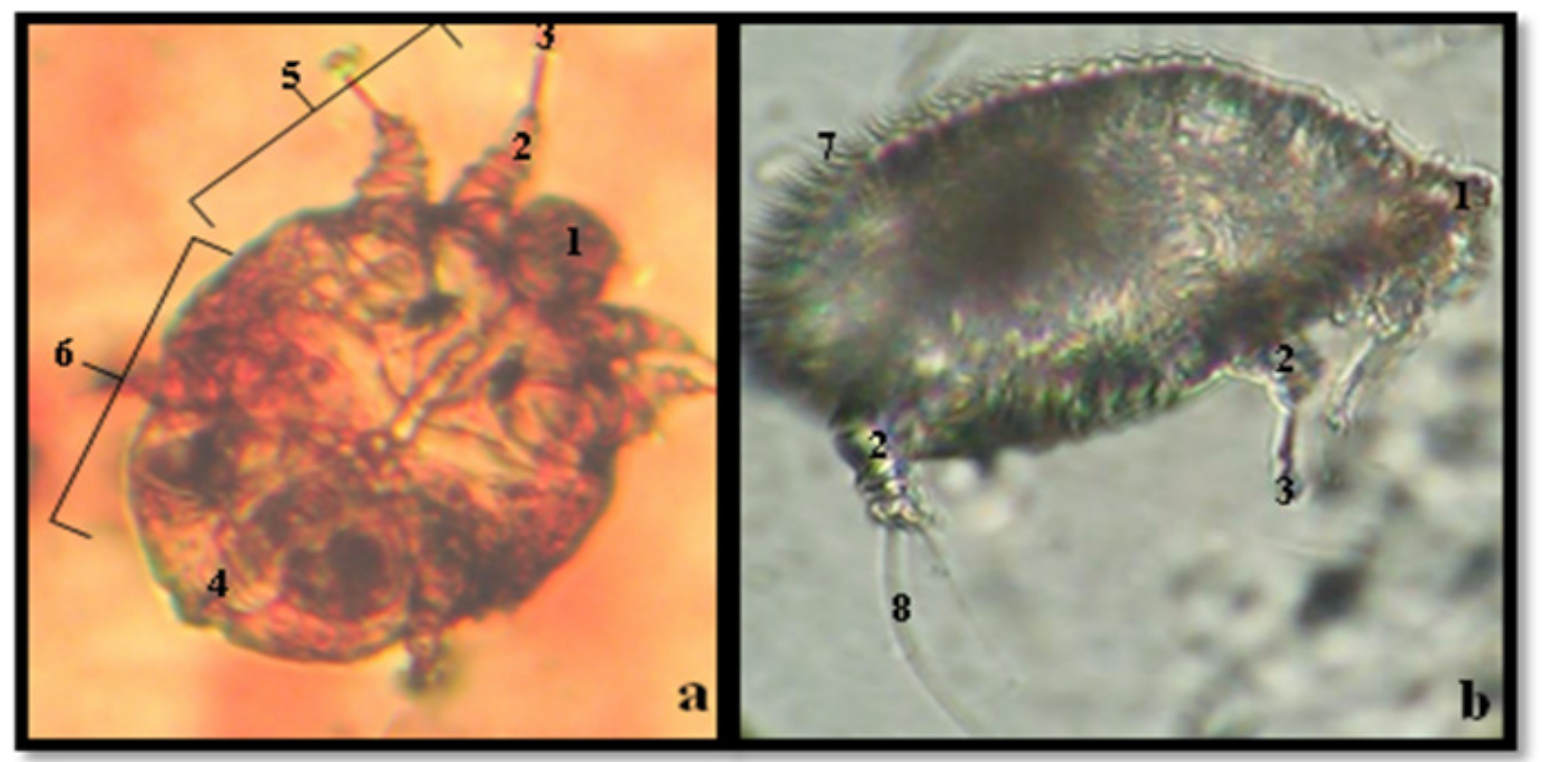

Figura 1. Trixacarus caviae adulto vista ventral y lateral. 1. Aparato bucal formada por quelíceros. 2. Cilindro quitinoso. 3. Ventosas cónicas. 4. Ano (ventral en la hembra). 5. Nototórax anterior (soporta los dos primeros pares de patas). 6. Notogáster posterior (los otros dos pares de patas). 7. Espinas. 8. Cerdas largas 
Las muestras se tomaron utilizando sacabocados y fueron colocadas en frascos de boca ancha, con solución de formol al 10\% para su fijación y traslado al laboratorio de patología animal para su proceso (corte $4 \mu \mathrm{m}$ y coloreadas con hematoxilina eosina). Se evaluaron los cambios histopatológicos de la piel en el mismo espécimen en los días 0,16 y 42 con la finalidad de relacionar las lesiones con los hallazgos histopatológicos y la evolución de las lesiones.

La evolución de las lesiones a causa del efecto acaricida fue clasificada en 3 categorías:

Categoría I: Leve desprendimiento de costras y recuperación parcial (10 - 40\%). Categoría II: Gran desprendimiento de costras y recuperación moderada (50 - 90\%) con crecimiento ligero de pelo.

Categoría III: Sin costras y recuperación completa $(100 \%)$ con crecimiento de pelo y capa homogénea.

A partir del conteo de ácaros se calculó el porcentaje promedio de ácaros vivos, tanto individualmente como en forma colectiva. También se determinó la efectividad del tratamiento mediante la siguiente fórmula:

Efectividad $=\left(\mathrm{N}^{\circ}\right.$ A. V. antes de la dosificación $-\mathrm{N}^{\circ}$ A. V. post dosificación) x 100

$\mathrm{N}^{\circ} \mathrm{A}$. V. antes de la dosificación

Dónde: A. V. = ácaros vivos

El efecto del tratamiento sobre el porcentaje de ácaros vivos post tratamiento se evaluó mediante la prueba de Kruskall Wallis y la diferenciación del tratamiento más eficiente se realizó utilizando pruebas consecutivas de U de Mann Whitney.

\section{RESULTADOS}

$\mathrm{Al}$ inicio del estudio, entre los animales tratados con fipronil, predominaron los animales con lesiones de grado 2 (dermatitis moderada); en el grupo que recibió ivermectina, predominaron las lesiones de grado 3 (dermatitis pronunciada). En el grupo control,

Tabla 1. Distribución de cuyes con sarna (T. caviae) según su presentación clínica y grados de lesión antes del tratamiento.

\begin{tabular}{lccc}
\hline \multirow{2}{*}{ Evaluación } & \multicolumn{3}{c}{$\mathbf{N}^{\circ}$ de animales/ Tratamiento } \\
\cline { 2 - 4 } & Fipronil $(\mathbf{n}=7)$ & Ivermectina $(\mathbf{n}=7)$ & Control $(\mathbf{n}=\mathbf{7})$ \\
\hline Presentación clínica & & & - \\
$\quad$ Leve & 6 & 6 & 5 \\
Moderada & - & - & 2 \\
Grave & & 1 & - \\
Lesiones & 1 & 3 & 3 \\
$\quad$ Grado 1: Dermatitis leve & 3 & 3 & 3 \\
Grado 2: Dermatitis moderada & 3 & - & 1 \\
Grado 3: Dermatitis pronunciada & - & & \\
Grado 4: Dermatitis costrosa & &
\end{tabular}

Tabla 2. Promedio de ácaros vivos de raspados cutáneos de cuyes con sarna (T. caviae), tratados con fipronil al $1 \%$ e ivermectina al $1 \%$

\begin{tabular}{ccccccccccc}
\hline & \multicolumn{3}{c}{ Fipronil } & \multicolumn{3}{c}{ Ivermectina } & \multicolumn{3}{c}{ Control } \\
Días & $\begin{array}{c}\text { Nro. } \\
\text { cuyes }\end{array}$ & $\begin{array}{c}\text { Total } \\
\text { ácaros }\end{array}$ & $\begin{array}{c}\text { Ácaros } \\
\text { vivos }\end{array}$ & $\%$ & $\begin{array}{c}\text { Total } \\
\text { ácaros }\end{array}$ & $\begin{array}{c}\text { Ácaros } \\
\text { vivos }\end{array}$ & $\%$ & $\begin{array}{c}\text { Total } \\
\text { ácaros }\end{array}$ & $\begin{array}{c}\text { Ácaros } \\
\text { vivos }\end{array}$ & $\%$ \\
\hline 0 & 7 & 21 & 21 & $100,0^{\mathrm{a}}$ & 20 & 20 & $100,0^{\mathrm{a}}$ & 22 & 22 & $100,0^{\mathrm{a}}$ \\
8 & 7 & 20 & 14 & $70,0^{\mathrm{b}}$ & 18 & 10 & $55,5^{\mathrm{b}}$ & 22 & 22 & $100,0^{\mathrm{a}}$ \\
16 & 7 & 18 & 8 & $44,4^{\mathrm{b}}$ & 17 & 5 & $29,4^{\mathrm{b}}$ & 22 & 22 & $100,0^{\mathrm{a}}$ \\
24 & 7 & 13 & 3 & $23,1^{\mathrm{b}}$ & 11 & 2 & $18,2^{\mathrm{b}}$ & 24 & 24 & $100,0^{\mathrm{a}}$ \\
32 & 7 & 10 & 1 & $10,0^{\mathrm{b}}$ & 9 & 0 & $0,0^{\mathrm{b}}$ & 26 & 26 & $100,0^{\mathrm{a}}$ \\
40 & 7 & 9 & 1 & $11,1^{\mathrm{b}}$ & 7 & 0 & $0,0^{\mathrm{b}}$ & 27 & 27 & $100,0^{\mathrm{a}}$ \\
\hline
\end{tabular}

ab Diferencia entre los grupos para cada periodo evaluado 
Tabla 3. Efectividad ( $\mathrm{N}^{\circ}$ de ácaros muertos post dosificación/\% efectividad) del fipronil al 1\% y la ivermectina al $1 \%$ en el tratamiento de sarna (T. caviae) en cuyes (C. porcellus)

\begin{tabular}{|c|c|c|c|c|c|c|c|c|c|c|c|c|}
\hline \multirow{2}{*}{ Tratamiento } & \multirow{2}{*}{$\begin{array}{c}\text { Dosis } \\
(\mathrm{ml} / \mathbf{K g})\end{array}$} & \multirow{2}{*}{$\begin{array}{c}\text { Ácaros } \\
\text { vivos al } \\
\text { inicio }\end{array}$} & \multicolumn{2}{|c|}{8 días } & \multicolumn{2}{|c|}{16 días } & \multicolumn{2}{|c|}{24 días } & \multicolumn{2}{|c|}{32 días } & \multicolumn{2}{|c|}{42 días } \\
\hline & & & $\mathbf{N}^{\circ}$ & $\%$ & $\mathbf{N}^{\circ}$ & $\%$ & $\mathbf{N}^{\circ}$ & $\%$ & $\mathbf{N}^{\circ}$ & $\%$ & $\mathbf{N}^{\circ}$ & $\%$ \\
\hline Fipronil & 1,5 & 21 & 7 & 33,3 & 13 & 61,9 & 18 & 85,7 & 20 & 95,2 & 20 & 95,2 \\
\hline Ivermectina & 0,05 & 20 & 10 & 50,0 & 15 & 75,0 & 18 & 90,0 & 0 & 100,0 & 0 & 100,0 \\
\hline
\end{tabular}

Tabla 4. Evolución de las lesiones a causa del efecto acaricida del fipronil al 1\% y la ivermectina al 1\% en el tratamiento de sarna (T. caviae) en cuyes (C. porcellus)

\begin{tabular}{|c|c|c|c|c|c|c|c|c|c|c|}
\hline \multirow{3}{*}{ Grado de lesiones } & \multicolumn{10}{|c|}{$\mathrm{N}^{\circ}$ animales recuperados en el proceso (días) } \\
\hline & \multicolumn{5}{|c|}{ Fipronil $1 \%$} & \multicolumn{5}{|c|}{ Ivermectina $1 \%$} \\
\hline & 8 & 16 & 24 & 32 & 42 & 8 & 16 & 24 & 32 & 42 \\
\hline $\begin{array}{l}\text { Leve desprendimiento de costras y } \\
\text { recuperación parcial }(10-40 \%)\end{array}$ & 3 & 2 & 2 & - & - & 3 & 2 & 1 & - & - \\
\hline $\begin{array}{l}\text { Gran desprendimiento de costras y } \\
\text { recuperación moderada ( } 50-90 \%)\end{array}$ & 2 & 3 & 1 & 2 & 1 & 2 & 2 & 2 & 1 & - \\
\hline $\begin{array}{l}\text { Sin costras y recuperación } \\
\text { completa }(100 \%)\end{array}$ & 2 & 2 & 4 & 5 & 6 & 2 & 3 & 4 & 6 & 7 \\
\hline
\end{tabular}

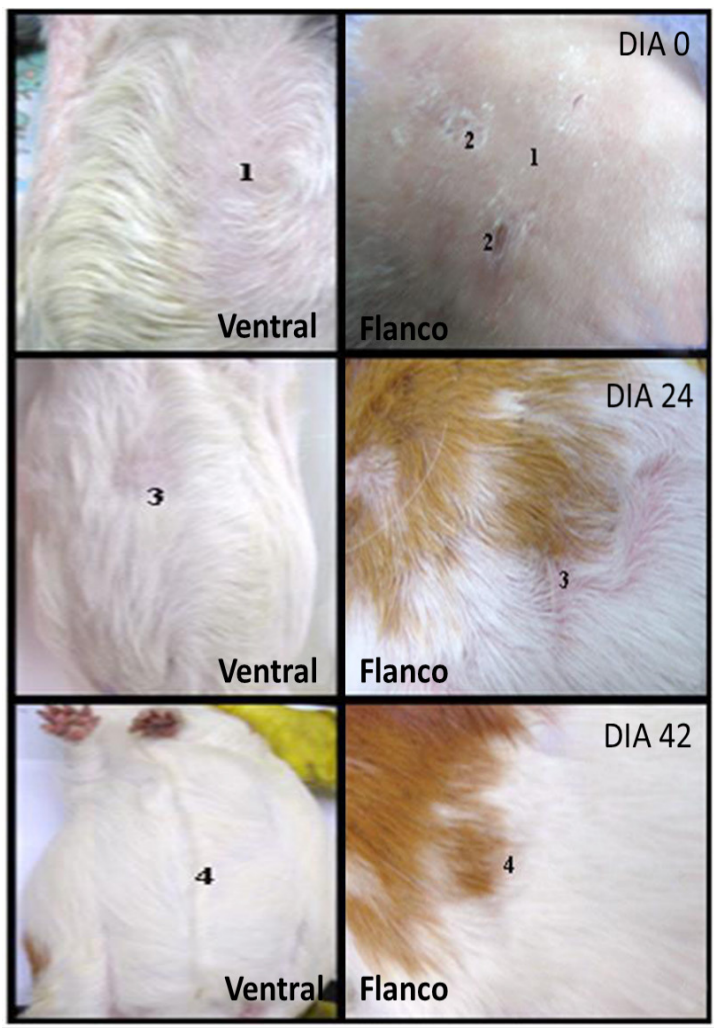

Día 0 : alopecia extendida y descamación de la piel (1 y 2)

Día 24: recuperación moderada con crecimiento ligero de pelo (3)

Día 42: recuperación completa y crecimiento abundante de pelo y capa homogénea (4)

Figura 2. Tratamiento Fiponil 1\%. Evolución de las lesiones a nivel ventral y de flanco. 


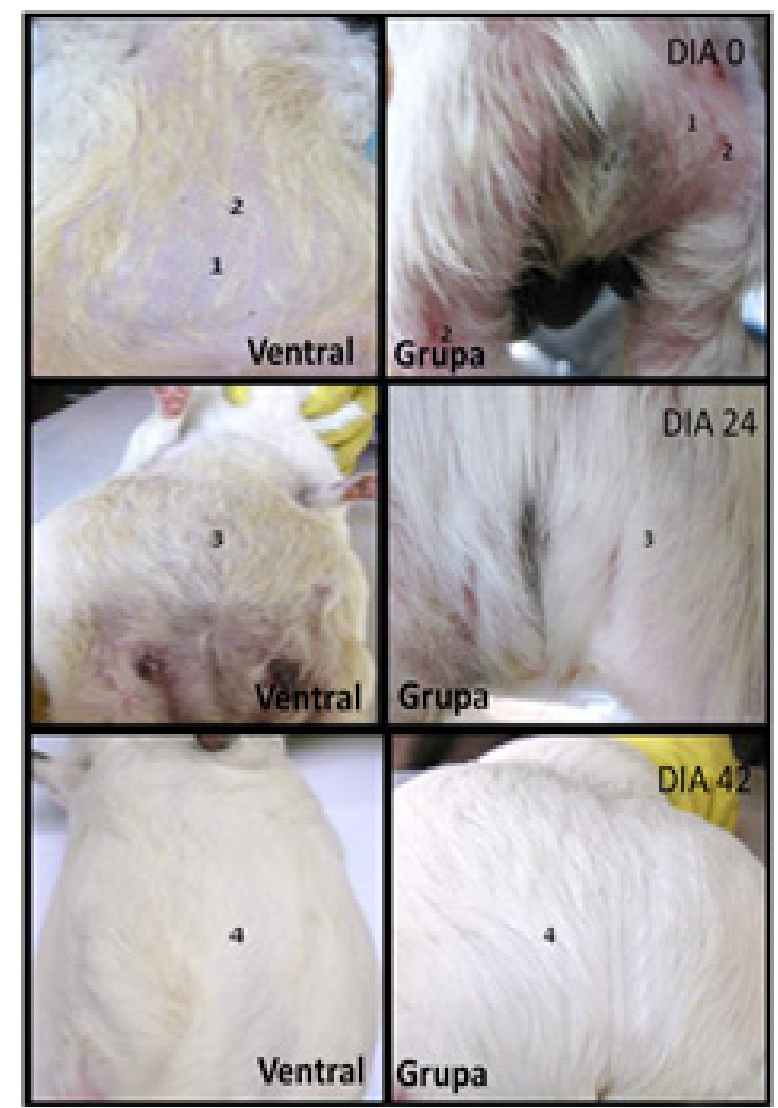

Día 0: alopecia extendida y descamación de la piel (1 y 2)

Día 24: recuperación moderada con crecimiento ligero de pelo (3)

Día 42: recuperación completa y crecimiento abundante de pelo y capa homogénea (4)

Figura 3. Tratamiento Ivermectina 1\%. Evolución de las lesiones a nivel ventral y de grupa.

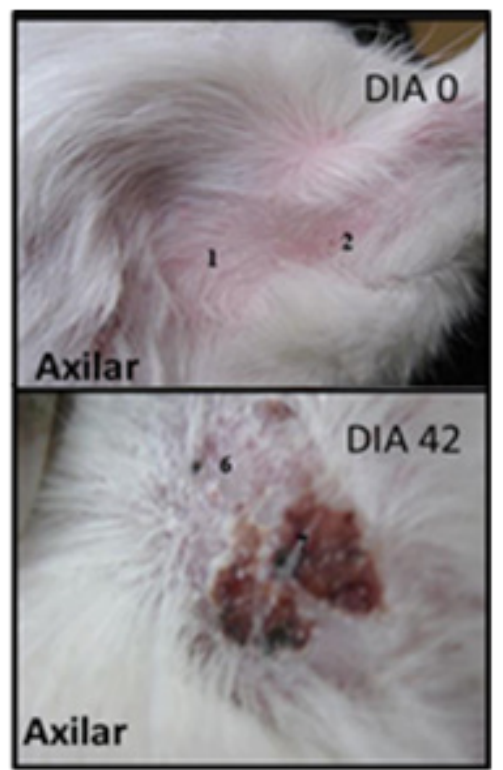

Día 0: alopecia focalizada, descamación, prurito y eritema en la zona axilar. (1 y 2 )

Día 42: alopecia extendida, descamación, gran cantidad de costras y ulceras abiertas (5 y 6).

Figura 4. Grupo Control sin tratamiento. Evolución de las lesiones a nivel axilar. algunos animales presentaron lesiones clínicas graves, mostrando lesiones de grado 4 (dermatitis costrosa), como se muestra en la tabla 1.

Los tres grupos inicialmente comenzaron con $100 \%$ de ácaros vivos. En el grupo tratado con fipronil, los animales presentaron una disminución progresiva hasta $11,1 \%$ en el día 42 que duró la evaluación, con respecto a los tratados con ivermectina que se observó una disminución en los porcentajes de ácaros vivos a $18,2 \%$ al día 24 y $0 \%$ en los días 32 y 42 . En el grupo control los ácaros vivos se mantuvieron en el 100\% durante todo el periodo experimental. Los detalles se presentan en la tabla 2 .

La medición de la efectividad del fipronil al 1\% alcanzó una efectividad del $95.2 \%$ en los días 32 y 42 del estudio. Con respecto a la ivermectina, la efectividad fue del $100 \%$ en los días 32 y 42 del estudio (tabla 3 ).

La tabla 4 presenta la evolución de las lesiones en los animales de los tres grupos de estudio. Entre 
los animales recuperados al día 42, en el grupo tratado con fipronil, un animal mantuvo recuperación corporal moderada del 80 - 90\% (encontrándose 1 ácaro vivo) y 6 animales presentaron un cuadro de recuperación completa con abundante crecimiento de pelo y capa homogénea (figura 2). En el grupo tratado con ivermectina el $100 \%$ de las lesiones presentaron recuperación completa en los 7 animales al día 42 de finalizado el estudio (figura 3). Las lesiones del grupo control avanzaron en extensión y gravedad durante el periodo experimental, mostrándose una dermatitis costrosa en diversas partes del cuerpo (figura 4).

El estudio histopatológico en el día 0 mostró una dermatitis eosinofílica con moderado infiltrado inflamatorio mononuclear difuso compuesto por

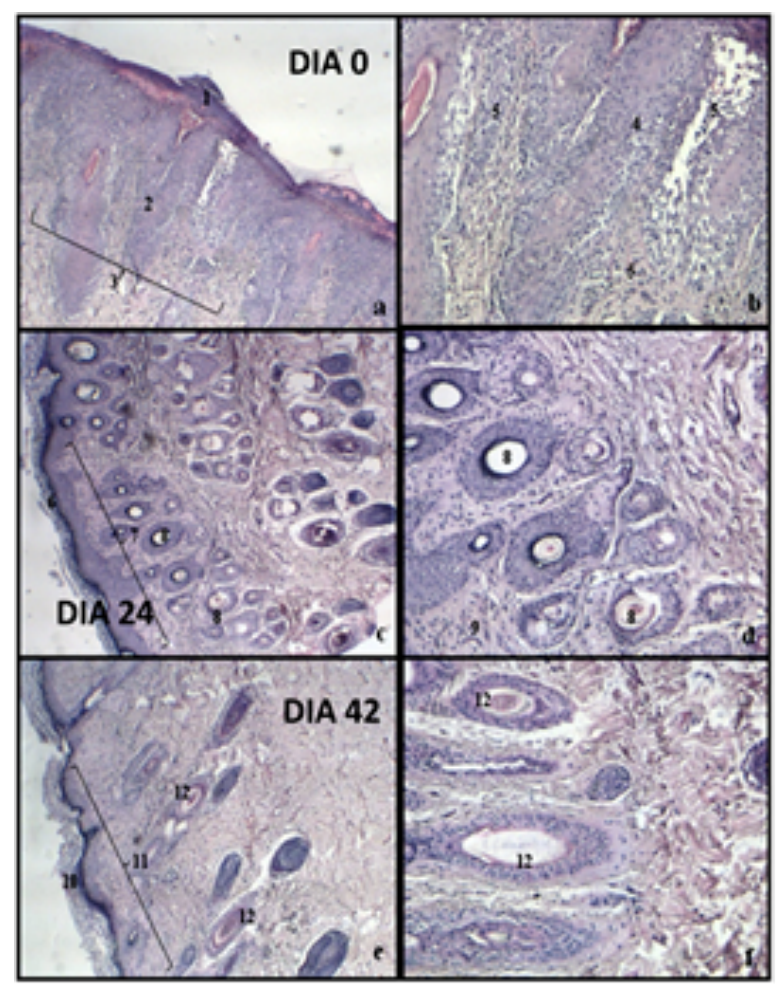

Día 0 : Histoarquitectura modificada

1. Hiperqueratosis paraqueratosica

2. Marcada hiperplasia de la epidermis.

3. Acantosis irregular

4. Moderada espongiosis

Día 24: Piel en regeneración

6. Moderada hiperqueratosis ortoqueratósica

7. Leve a moderada acantosis irregular

8. Folículos pilosos viables y no viables

9. Leve infiltrado inflamatorio mononuclear focal a nivel de la dermis superficial.

Día 42: Regeneración de la histoarquitectura

10. Leve hiperqueratosis ortoqueratósica

11. Ausencia de acantosis y espongiosis

12. Folículos pilosos viables. H.E. X 10 y 40 .

Figura 5. Evolución histopatológica de la piel. macrófagos, linfocitos y eosinófilos a nivel de la dermis superficial en los tres grupos, esta histoarquitectura se mantuvo en todos los períodos de evolución del grupo control (figura 5 (a y b)). Al día 24, se observó folículos pilosos viables y no viables y leve infiltrado inflamatorio (figura 5 ( c y d)). La evolución del proceso de recuperación histológica en los grupos fipronil e ivermectina fue similar. A los 42 días la piel no mostró cuadro inflamatorio, observándose signos de regeneración a nivel epidérmico y reorganización a nivel dérmico (figura 5 (e y f)).

El análisis estadístico indica que no existe diferencia significativa en porcentaje de efectividad de las drogas entre los animales tratados con fipronil e ivermectina, pero sí de estos con respecto al grupo control $(\mathrm{p}<0,05)$.

\section{DISCUSIÓN}

El fipronil al 1\% demostró ser eficaz en su acción acaricida. La aplicación en dosis repetidas fue sugerida, a raíz de los resultados de un estudio piloto (Levano, comunicación personal). El efecto se observo a partir del día 24, a diferencia de los resultados obtenidos por Titi (2000) quien utilizó fipronil al 1\% en una sola dosis de $1 \mathrm{ml} / 10 \mathrm{~kg}$ p.v. en alpacas, obteniendo un porcentaje promedio de ácaros vivos de $12,7 \%$ y $0 \%$ a los 7 y 14 días post tratamiento, respectivamente. Las diferencias se deben probablemente al metabolismo de la droga, a mayor tamaño corporal el metabolismo se hace más lento y a menor tamaño corporal, el metabolismo se hace más rápido; por lo tanto, las reacciones metabólicas en cuyes ocurren a mayor velocidad que en otros animales (Sumano y Ocampo, 1997; Adams, 2001; Botana et al., 2002; Plumb, 2005).

La ivermectina al $1 \%$ en dosis de $0,05 \mathrm{ml} / \mathrm{kg}$ p.v., también tuvo resultados positivos en el tratamiento de $T$. caviae utilizando el mismo esquema de aplicación del fipronil. A partir del día 8 se observó una disminución considerable en la cantidad de ácaros en los raspados, y a partir del día 24 hasta el 42 que duró el estudio, no se observó la presencia de ácaros vivos. Portocarrero, Chavez, Falcon y Chavera (1998), y Valencia y Castrejón (2000) trabajaron con ivermectina al $1 \%$ en estudios en alpacas y Bazán (1989) en sarna de conejos. En todos los casos no se evidenciaron ácaros vivos entre 35 y 60 días post tratamiento, esto se debería a que la droga posee una concentración máxima en el plasma de 5 a 7 días y el tiempo de liberación desde la grasa es 7 días (Botana et al., 2002), por lo que la elevada afinidad por los lípidos 
facilita su depósito, principalmente en el hígado y la grasa, lo cuales actúan como un reservorio del fármaco relevante para la persistencia de la actividad antiparasitaria contra el parásito.

En este estudio la efectividad del fipronil fue 95,2 $\%$ a partir del día 32 hasta el final el estudio. Esto no sucede en estudios realizado en otras especies. Por ejemplo, Titi (2000) trabajó con un grupo de alpacas con sarna usando fipronil al $1 \%$ a dosis de $1 \mathrm{ml} / 10 \mathrm{~kg}$ y Curtis (1996) utilizando fipronil al $0,25 \%$ en perros en tres dosis consecutivas de $3 \mathrm{ml} / \mathrm{kg}$ p.v., en ambos casos se observó una efectividad del $100 \%$ a partir del día 14 post tratamiento. Estos resultados comparados con el presente estudio se deben probablemente a que los diferentes parásitos, incluyendo los ácaros, poseen variada susceptibilidad frente a la acción de los fármacos y al metabolismo de la droga en cada especie animal.

En el caso de la efectividad de la ivermectina se obtuvo $100 \%$ al día 32 , lo que resulta similar a otros estudios como Alva y Franco (1995), Portocarrero et al., (1998) y Chávez, Casas y Suni (2000), quienes trabajaron con diferentes especies y utilizaron ivermectina al $1 \%$ en una sola dosis de $0,02 \mathrm{ml} / \mathrm{kg}$ p.v.

La dosis de fipronil empleada para el estudio corresponde a lo obtenido como resultado positivo al tratamiento de $T$. caviae en un estudio piloto realizado por Levano y Chauca (2008). Sin embargo, la determinación de una dosis mínima efectiva y el poder residual puede ser motivo de otra investigación.

La necesidad de aplicar más de una dosis con ambos productos está relacionada al fracaso obtenido al tratamiento con dosis única. Este fracaso puede estar asociado al comportamiento y ciclo de vida del parásito (Swindle et al., 1996; Richardson, 2000; White et al., 2003; Paterson, 2006), sostienen que el ciclo de vida del T. caviae es 14 días. Sin embargo Fuentealba y Hanna (1996) mencionan que el ciclo de vida del parásito es aún desconocido y que este varía de acuerdo al lugar y las condiciones climáticas. Hasta el momento no se ha reportado en el Perú estudios sobre el ciclo de vida del $T$. caviae. Por ello, Richardson (2000), White et al., (2003), Beck y Pantchev (2012), y Paterson (2006) recomiendan usar de tres a más aplicaciones consecutivas de ivermectina. Así mismo, no existen reportes del efecto acaricida del fipronil, por lo cual se instituyó un protocolo de tratamiento de cuatro aplicaciones consecutivas. Esto debido a que ambos tratamientos no afectan los huevos, sino las formas activas juveniles y adultas, por lo cuál la aplicación de cuatro dosis consecutivas permite la persistencia en la sangre de los productos sistémicos (ivermectina), o el poder residual de una droga que actúe por contacto (fipronil), cubriendo el máximo tiempo para asegurar la eliminación de larvas recién eclosionadas provenientes de un huevo sobreviviente (Botana et al., 2002).

La mitad de los animales tratados con fipronil al $1 \%$ e ivermectina al $1 \%$ que presentaron lesiones clínicas de grado 1 a 2 (dermatitis leve a moderada) mostraron la piel con recuperación de moderada $(50-90 \%)$ a completa $(100 \%)$ entre los 16 y 24 días post tratamiento. Estos resultados son similares a los obtenidos por Alva y Franco (1995), Portocarrero et al., (1998) y Chávez et al., (2000) quienes usando ivermectina al $1 \%$ encontraron desprendimiento de costras y la recuperación completa de la piel en más de la mitad de los animales entre los días 15 y 30 post tratamiento. No obstante, en el estudio partir del día 32 hasta el día 42 que duró el estudio todos los animales tratados con fipronil e ivermectina mostraron una piel completamente recuperada y sin costras (100\%). En el grupo tratado con fipronil, solamente un cuy presentó un ácaro vivo, en una piel sana (90\%), lo que permite deducir que su hallazgo fue accidental. No se presentaron reacciones adversas secundarias como toxicidad posterior a la aplicación de los medicamentos empleados en el estudio.

En esta investigación, los cambios histopatológicos encontrados en la piel de cuyes afectados con T. caviae se describen de similar forma que los encontrados en otras investigaciones. Se confirman la presencia de hiperqueratosis ortoqueratósica e hiperplasia epidérmica las que son características de las modificaciones crónicas de la epidermis. Las lesiones de fibrosis y acantosis de la epidermis, zonas de necrosis y edema, son mencionadas con la presencia de eosinófilos y linfocitos, que parece ser característico de la reacción inflamatoria de la piel frente al ácaro (Fuentealba y Hanna, 1996; Portocarrero et al.,1998).

Dorrestein y Van Bronswijk (1979) en Australia, no reportaron la presencia de ácaros en algunos cortes histopatológicos, resultado que coincide con el estudio realizado. Esto puede deberse a que estos ácaros pueden estar en la superficie de la piel a nivel del estrato corneo, el cual al momento del manejo de la zona indicada para la biopsia, puede salir al medio exterior. El uso de cortes seriados en el diagnóstico histopatológico podría ayudar a evidenciar la presencia 
del parásito, metodología que no estuvo considerada en el estudio.

Los resultados del estudio demuestran la efectividad contra $T$. caviae de las dos drogas utilizadas, mediante diferentes vías de aplicación. El fipronil aplicado vía epicutánea, hace que su absorción sea más lenta, difundiéndose en todas las estructuras ricas en lípidos de la epidermis: estrato córneo, estrato germinativo, grasa perifolicular y complejo pilo sebáceo (Adams, 2001). En el caso de la ivermectina la aplicación vía subcutánea permite su depósito en el espacio subcutáneo, lo cual favorece a una absorción lenta, manteniéndose en la circulación sistémica en una concentración mínima eficaz, evitando la sobrevivencia de los ácaros (Botana et al, 2002). Esto se debe al tipo de vehículo de la droga, es decir a los excipientes que permiten una liberación lenta pero con niveles apropiados para un efecto acaricida prolongado (Botana et al., 2002; Plumb, 2005).

Por otro lado, la mayor practicidad en el uso de las drogas se le atribuye al fipronil, debido a la manipulación del animal y su aplicación topical que requiere de una sola persona (ahorro en mano de obra). A diferencia de la ivermectina, donde la ayuda de otra persona especializada para realizar el manejo y la aplicación subcutánea del producto es indispensable.

Finalmente, se debe tener en cuenta que el control de la sarna en cuyes causada por $T$. caviae debe de incluir un adecuado control sanitario, donde la educación sanitaria a los productores es importante, el conocimiento de la biología del ácaro, el hacer uso racional y alternativo de los acaricidas existentes y conocer la eficacia de ellos, el poder residual y el costo/beneficio de los productos a utilizar.

\section{CONCLUSIONES}

Se concluye que el Fipronil al $1 \%$ a dosis $1,5 \mathrm{ml} /$ $\mathrm{Kg}$. p.v. por vía epicutánea y la Ivermectina al $1 \%$ a dosis de $0,05 \mathrm{ml} / \mathrm{kg}$ p.v. por vía subcutánea, aplicados en cuatro dosis consecutivas con intervalos de 8 días mostraron un efecto acaricida sobre el $T$. caviae. La recuperación clínica de la piel afectada por $T$. caviae en animales tratados con fipronil al $1 \%$ y ivermectina al $1 \%$ fue evidente desde los 24 días post tratamiento.

\section{Correspondencia:}

Meylin Huamán Alcantara

Correo electrónico: mhuamana@inia.gob.pe

\section{REFERENCIAS BIBLIOGRÁFICAS}

1. Ackerman, L. (1987). Trixacarus caviae infestation in a guinea Pig. Rev Can Vet, 28, 613

2. Adams, H. R. (2001). Veterinary pharmacology and therapeutics. ( $8^{\text {th }}$ Edition). Ames: Iowa State Univ.

3. Alva, J. \& Franco, E. (1995). Seguimiento de efectividad de los medicamentos sistémicos (Cydectin, Ivomec, Ivermec y Dectomax) en el control de sarna en alpacas. Rev Cienc Vet, Perú, 11, $18-21$.

4. Bazán, J. (1989). La ivermectina en el tratamiento de la sarna sarcóptica (Sarcoptes scabiei var. Cuniculi) en conejos. (Tesis de Médico Veterinario. Universidad Nacional de Cajamarca, Cajamarca, Perú).

5. Beck, W. \& Pantchev, N. (2012). Praktische Parasitologie bei Heimtieren: Kleinsäuger-VögelReptilien-Bienen. Hannover: Schlütersche.

6. Botana, L. M., Landoni, M. F., \& Martín-Jiménez, T. (2002). Farmacología y terapéutica veterinaria. Madrid: McGraw-Hill Interamericana.

7. Collins, G. H., Pope, S., \& Griffin, D. L. (1986). Trixacarus caviae Fain et al.(Acari: Sarcoptidae): Dimensions, population composition and development of infection in guinea pigs. Australian Journal of Entomology, 25(1), 17-22.

8. Curtis, C. F. (1996). Use of 0.25 per cent fipronil spray to treat sarcoptic mange in a litter of five-weekold puppies. Vet Rec, 139, 43-44.

9. Chávez, A., Casas, E., \& Suni, S. (2001). Evaluación de la efectividad y el efecto residual de una ivermectina simple y una ivermectina de larga acción (Trust LA), contra sarna sarcóptica en alpacas. $M V$ Rev Cien Vet, 17(2), 33-34.

10. Dorrestein, G. M. \& Van Bronswijk, J. E. M. H. (1979). Trixacarus caviae Fain, Howell \& Hyatt 1972 (Acari: Sarcoptidae) as a cause of mange in guinea-pigs and papular urticaria in man. Veterinary Parasitology, 5(4), 389-398.

11. Fuentealba, C., \& Hanna, P. (1996). Mange induced by Trixacarus caviae in a guinea pig. The Canadian Veterinary Journal, 37(12), 749.

12. Hendrix, Ch. (1999). Diagnóstico parasitológico veterinario. (Segunda edición, p 209-210.). Madrid: Mosby.

13. Klompen, J. (1992). Phylogenetic relationships in the mite family Sarcoptidae (Acari: Astigmata). (p. 29-33). Michigan: Museum of Zoology, University of Michigan.

14. Levano, M. \& Chauca, L. (2008). Identificación del Trixacarus caviae en granjas de cuyes familiar y familiar comercial investigación participativa, estudio de caso. XXXI Reunión APPA, Lima, Perú.

15. Paterson, S. (2006). Skin Diseases of Exotic Pets. (2nd edition, p 237-238). Australia: Blackwell. 
16. Plumb, D. (2005). Veterinary Drug Handbook. (5th edition). USA: Blackwell.

17. Portocarrero, M., Chavez, A., Falcon, N., \& Chavera, A. (1998). Efecto residual de la Ivermectina LA en el control de la sarna sarcoptica de alpacas y sus cambios histopatologicos en piel. Rev Inv Pec IVITA (Peru), 9, 34-40

18. Richardson, V. (2000). Diseases of domestic guinea pig. (2nd edition, p 3-4). Australia: Blackwell Science.

19. Scott, D., Miller, W., Griffin, C., Giovanniello, O. \& Jure, A. (2002). Muller \& Kirk's: dermatología en pequeños animales. Buenos Aires : Intermédica.

20. Sumano, H. \& Ocampo, L. (1997). Farmacología veterinaria. ( $2^{\mathrm{a}}$ edición, pp. 277-280). Ciudad de Mexico: Mc Graw-Hill.
21. Swindle, M., Laber-Laird, K. \& Flecknell, P. (1996). Handbook of Rodent and Rabbit Medicine. (pp. 119120). Oxford: PEGAMON.

22. Titi, J. (2000). Efecto del Fipronil en el Control de Ectoparásitos de Alpacas (Lama pacos). (Tesis de Médico Veterinario y Zootecnista. Universidad Nacional del Altiplano, Puno, Perú).

23. Valencia, N. \& Castrejón, M. (2000). Evaluación antisárnica y piojicida de una ivermectina de larga acción en alpacas. Rev Cienc Vet Perú, 16, 26-28.

24. White, S. D., Bourdeau, P. J., \& Meredith, A. (2003). Dermatologic problems in guinea pigs. Compendium, 25(9), 690-700. 